\title{
Construction of disease-specific transcriptional regulatory networks identifies co-activation of four gene in esophageal squamous cell carcinoma
}

\author{
YU ZHAO* , LI MIN* , CHANGQIN XU, LINLIN SHAO, SHUILONG GUO, RUI CHENG, \\ JIE XING, SHENGTAO ZHU and SHUTIAN ZHANG \\ Department of Gastroenterology, Beijing Friendship Hospital, Capital Medical University, \\ National Clinical Research Center for Digestive Disease, Beijing Digestive Disease Center, \\ Beijing Key Laboratory for Precancerous Lesions of Digestive Disease, \\ Xicheng, Beijing 100050, P.R. China
}

Received October 11, 2016; Accepted February 2, 2017

DOI: $10.3892 /$ or.2017.5681

\begin{abstract}
Even though various molecules may serve as biomarkers, little is known concerning the mechanisms underlying the carcinogenesis of ESCC, particularly the transcriptional regulatory network. Thus, in the present study, paired ESCC and non-cancerous (NC) tissues were assayed by Affymetrix microarray assays. Passing Attributes between Networks for Data Assimilation (PANDA) was used to construct networks between transcription factors (TFs) and their targets. AnaPANDA program was applied to compare the regulatory networks. A hypergeometric distribution model-based target profile similarity analysis was utilized to find co-activation effects using both TF-target networks and differential expression data. There were 1,116 genes upregulated and 1,301 genes downregulated in ESCC compared with NC tissues. In TF-target networks, 16,970 ESCC-specific edges and 9,307 NC-specific edges were identified. Edge enrichment analysis by AnaPANDA indicated 17 transcription factors (NFE2L2, ELK4, PAX6, TLX1, ESR1, ZNF143, TP53, REL, ELF5, STAT1, TBP, NHLH1, FOXL1, SOX9, STAT3, ELK1, and HOXA5) suppressed in ESCC and 5 (SPIB, BRCA1, MZF1, MAFG and NFE2L1) activated in ESCC. For SPIB, MZF1, MAFG and NFE2L1, a strong and significant co-activation effect among them was detected in ESCC. In conclusion, the construction of transcriptional regulatory networks found
\end{abstract}

Correspondence to: Professor Shutian Zhang or Dr Shengtao Zhu, Department of Gastroenterology, Beijing Friendship Hospital, Capital Medical University, National Clinical Research Center for Digestive Disease, Beijing Digestive Disease Center, Beijing Key Laboratory for Precancerous Lesions of Digestive Disease, 95 Yong-An Road, Xicheng, Beijing 100050, P.R. China

E-mail: zhangshutian@ccmu.edu.cn

E-mail: shengtaozhu@126.com

*Contributed equally

Key words: network analysis, transcriptional factors, ESCC
SPIB, MZF1, MAFG and NFE2L1 co-activated in ESCC, which provides distinctive insight into the carcinogenesis mechanism of ESCC.

\section{Introduction}

Esophageal cancer (EC) is the sixth leading cause of cancer-related mortality and the eighth most frequently diagnosed cancer, worldwide. There are 481,000 new cases of esophageal cancer each year, which also indicates a rapidly increasing incidence rate $(1,2)$. Based on histology, esophageal cancer can be mainly categorized into esophageal squamous cell carcinoma (ESCC) and esophageal adenocarcinoma (EAC), which accounts for $\sim 90 \%$ of all esophageal cancers. Compared with EAC, ESCC has a higher incidence worldwide, particularly in the so-called Asian belt (Turkey, Northeastern Iran, Kazakhstan and Northern and Central China), where ESCC has been noted to account for $\sim 90 \%$ of the total EC cases (3). Such as other carcinomas, ESCC is likewise a complex and heterogeneous disease.

Molecular markers in EC patients have been widely studied, and a meta-analysis summarized the results of published studies regarding the prognostic role (4), including 109 studies for 13 different markers. It showed that VEGF, cyclin D1, Ki-67 and squamous cell carcinoma antigen can be used in ESCC, and COX-2, HER-2 in EAC as prediction markers for overall survival. In the early event of ESCC, DNA hypermethylation is frequently found in promoter regions, and some molecular markers such as DAPK, p16, MGMT, MLH1, RAR $\beta 2$, HIN1, TFPI-2, DACH1 and SOX17 were found methylated in the precursor lesions of human esophageal epithelia (5-10). Others, such as CDH1, RASSF1A, P16 and FHIT were found to be involved in tumor progression and poor prognosis (11-15). Even though some molecules may serve as biomarkers, little is known concerning the carcinogenesis mechanism of ESCC, particularly the transcriptional regulatory network. Thus, more integrative, systems-level analyses are necessary in order to better understand how ESCC develops and progresses, and how it may respond to different therapeutic interventions. 
Gene expression profiling assay has been used to explore the molecular mechanisms in various types of tumors, which provides a powerful approach to examine the expression profiles of virtually all known genes at once (16-19). However, profiling analyses only detect differentially expressed genes and provide limited insight into the underlying mechanism. More information could be obtained only through integrated analysis combining data from different dimensions.

Passing Attributes between Networks for Data Assimilation (PANDA) is an integrative network inference method based on a message-passing approach $(20,21)$. It defines transcription factors (TFs) as transmitters and their target genes as receivers. By integrating mRNA expression data with the TF motif data, which link TFs with their potential targets, it generates a $\mathrm{Z}$-score reflecting the confidence level of regulatory relationship for each TF-target edge. PANDA has been successfully used to study several diseases including chronic obstructive pulmonary disease (COPD), emphysema and ovarian cancer

In the present study, we applied PANDA for constructing and comparing regulatory networks in ESCC and its paired non-cancerous (NC) tissues, and integrate this information with differential expression data to obtain a better understanding of the carcinogenesis mechanism of ESCC, as well as identification of new biomarkers.

\section{Materials and methods}

Tissue sample handling. For GeneChip analysis, ESCC and paired normal esophageal mucosa samples were obtained from 8 ESCC patients who underwent esophagectomy at Beijing Friendship Hospital from September to December 2012. The median age of the patients when diagnosed was 63 years (range, 46-73 years). Two of the 8 ESCC patients were men, and 6 were women. Well-differentiated, moderately differentiated, poorly differentiated ESCC were found in 1, 4 and 3 cases, respectively (more details are shown in Table I). Another 5 ESCC tissues from patients who underwent esophagectomy at Beijing Friendship Hospital from November 2016 to January 2017 were recruited as a validation cohort. Study protocols were approved by the Ethics Committee of the Affiliated Hospital of Capital Medical University, and all experiments were performed in accordance with approved guidelines of the Affiliated Hospital of Capital Medical University. Written informed consent was obtained from the patients for publication of the present study and any accompanying images.

Isolation and GeneChip analysis. Total RNA extraction, quality detection, GeneChip assay protocols as well as preliminary analysis reports of differentially expressed gene were described in our previous study (22). All CEL raw data were background corrected by the RMA method. Then, $\log 2$ transformation along with quantile normalization were also subsequently applied.

TF-target network construction and comparison. We used PANDA models to evaluate the regulatory relationship between TFs and their targeted genes in both ESCC and NC tissues (Fig. 1). A 'prior' regulatory network by mapping transcription factor motifs to a reference genome was derived from JASPAR database. For protein-protein interactions
Table I. Demographic and pathological characteristics of the 8 ESCC patients.

\begin{tabular}{lcclll}
\hline $\begin{array}{l}\text { Pt. } \\
\text { no. }\end{array}$ & Sex & Age & Differentiation & $\begin{array}{c}\text { TNM } \\
\text { stage }\end{array}$ & $\begin{array}{c}\text { AJCC } \\
\text { stage }\end{array}$ \\
\hline 1 & M & 63 & Poor & T3N1M0 & III \\
2 & M & 72 & Moderate & T3N0M0 & IIA \\
3 & M & 46 & Moderate & T1N0M0 & I \\
4 & M & 55 & Moderate & T1N1M0 & IIA \\
5 & M & 59 & Well & T1N0M0 & I \\
6 & M & 51 & Poor & T2N1M0 & IIB \\
7 & F & 68 & Moderate & T4N1M0 & III \\
8 & F & 53 & Poor & T3N1M0 & III \\
\hline
\end{tabular}

ESCC, esophageal squamous cell carcinoma; Pt., patient; TNM, tumor-node-metastasis; AJCC, American Joint Committee on Cancer; $\mathrm{M}$, male; F, female.

(PPI), a publicly available dataset was used as an estimate. Then, subnetworks of edges that are most distinct between each pair of our reconstructed network models were identified by AnaPANDA program (details are shown in the Results section).

Co-activation and co-repression analysis. ESCC- and $\mathrm{NC}$-specific edges were respectively extracted for co-activation and co-repression analysis. All extracted edges linked with a differentially expressed target were selected for further analysis. For each 2-paired TFs, a Fisher's exact test was performed and a p-value was calculated to evaluate ESCC-specific co-activation effects and $\mathrm{NC}$-specific co-activation effects (ESCC-specific co-repression effects).

$R T-q P C R$ validation of gene expression. Five ESCC and paired $\mathrm{NC}$ tissues of the validation cohort were handled in the same way as microarray assays, as previously described (22). cDNA was synthesized from $\sim 1 \mu \mathrm{g}$ RNA and qPCR settings were $94^{\circ} \mathrm{C}$ for $2 \mathrm{~min}$ followed by 40 cycles of $94^{\circ} \mathrm{C}$ for $15 \mathrm{sec}, 60^{\circ} \mathrm{C}$ for $20 \mathrm{sec}$ and $72^{\circ} \mathrm{C}$ for $30 \mathrm{sec}$, and then followed by $72^{\circ} \mathrm{C}$ for $2 \mathrm{~min}$.

Statistical analysis and data visualization. All statistical tests were performed using R 3.3.1 software (www.r-project. org). All statistical tests were two-tailed and $\mathrm{p}<0.05$ was considered statistically significant. Venn diagram, ggplot2 and pheatmap $\mathrm{R}$ packages were used for data visualization.

\section{Results}

Building TF-target regulatory networks of ESCC and NC tissues. ESCC and paired NC samples were obtained from 8 ESCC patients who underwent esophagectomy. mRNA was extracted and gene profiling data were obtained. Firstly, gene expression differences between ESCC and NC were evaluated by paired t-test, and a cut-off of FDR adjusted $p<0.05$ was used to determine significant differentially expressed genes. There were 1,116 upregulated genes and 1,301 downregulated genes in ESCC compared with NC (Fig. 2A). Then, TF-target 


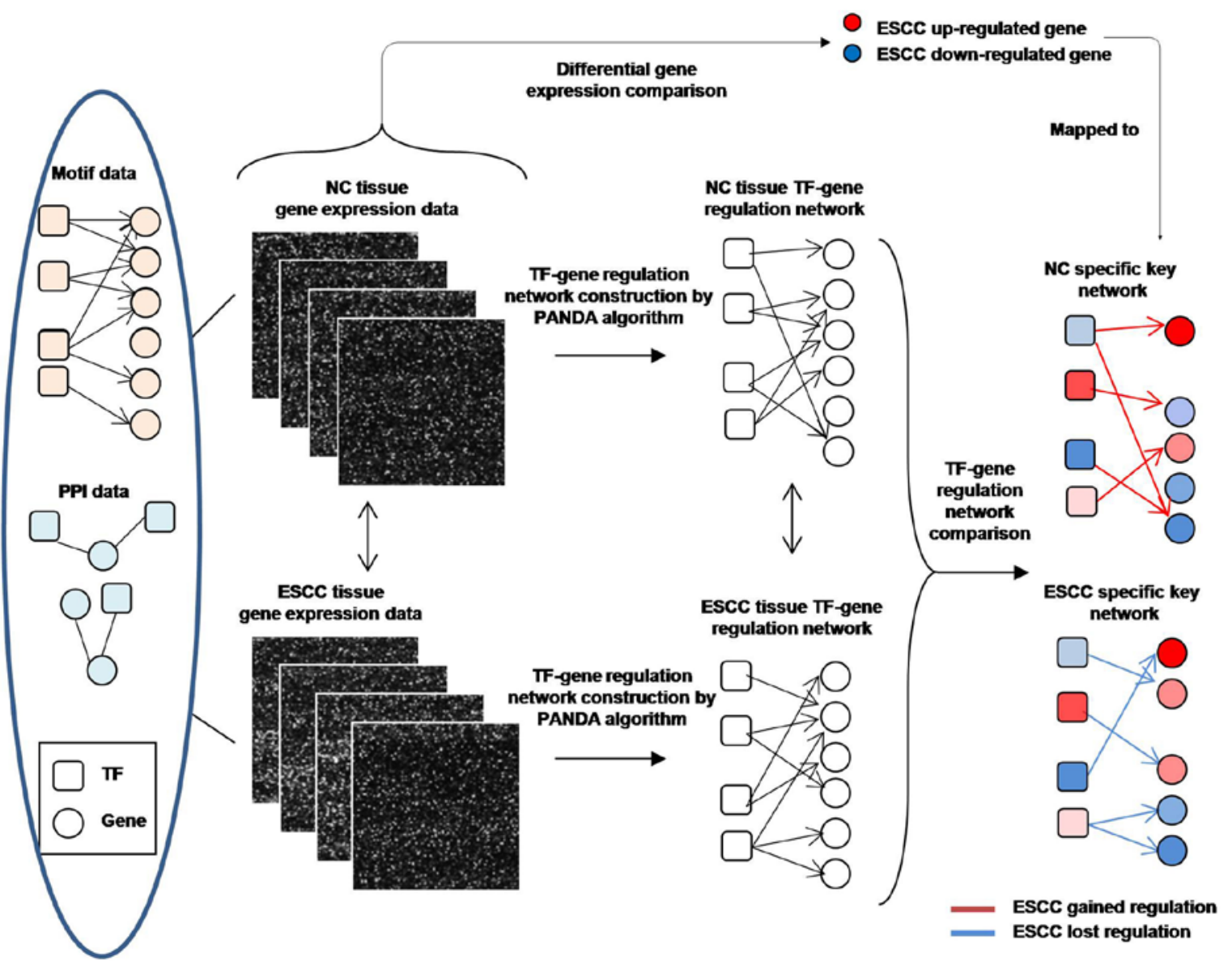

Figure 1. An outline of how we integrate PANDA network analysis with differential gene expression analysis. Firstly, TF-target regulatory networks were constructed by PANDA integrating gene expression data with motif and PPI data. Secondly, networks of esophageal squamous cell carcinoma (ESCC) and non-cancerous (NC) tissues were compared. Finally, differentially expressed genes were mapped to these networks and TFs with a significant activity change were enriched by AnaPANDA. TF, transcription factor.

A

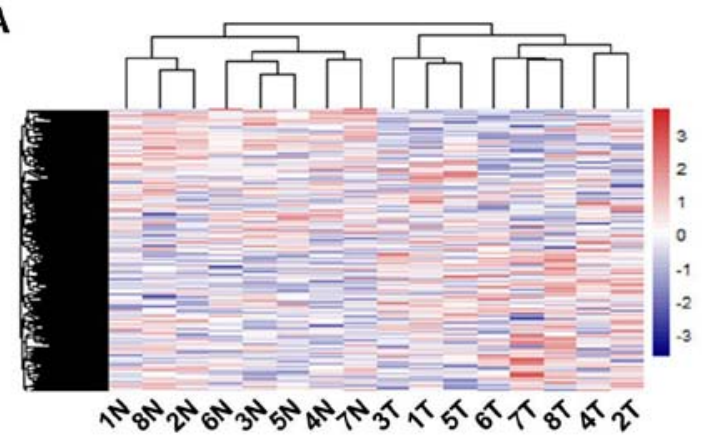

C

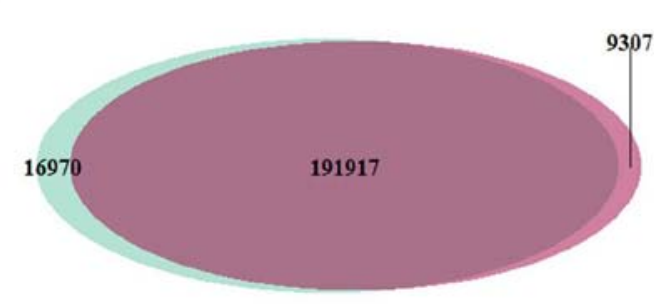

ESCC TF-gene edges
B

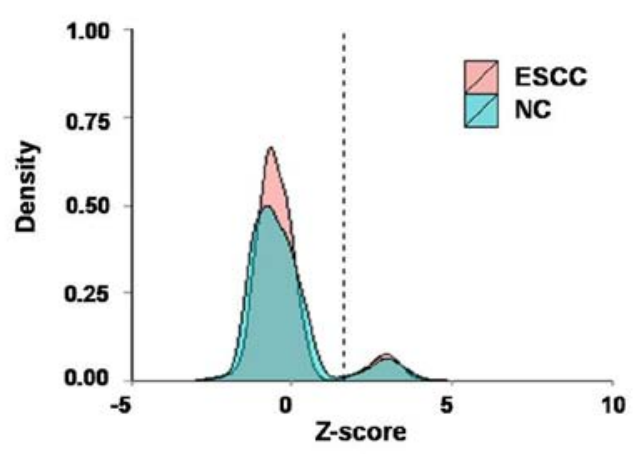

D

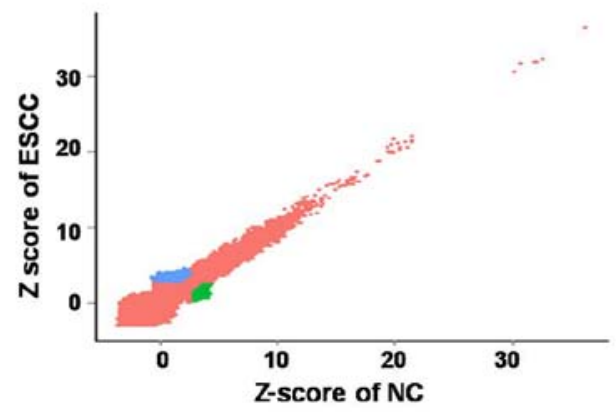

Figure 2. Construction of TF-target regulatory networks of squamous cell carcinoma (ESCC) and non-cancerous (NC) tissues. (A) Differentially expressed genes in ESCC and NC tissues. (B) Z score distribution of TF-target edges in networks of ESCC and NC tissues. (C) Venn diagram of edge overlapping between ESCC and NC. (D) NC- and ESCC-specific edges filtering based on Z-scores. TF, transcription factor. 
A

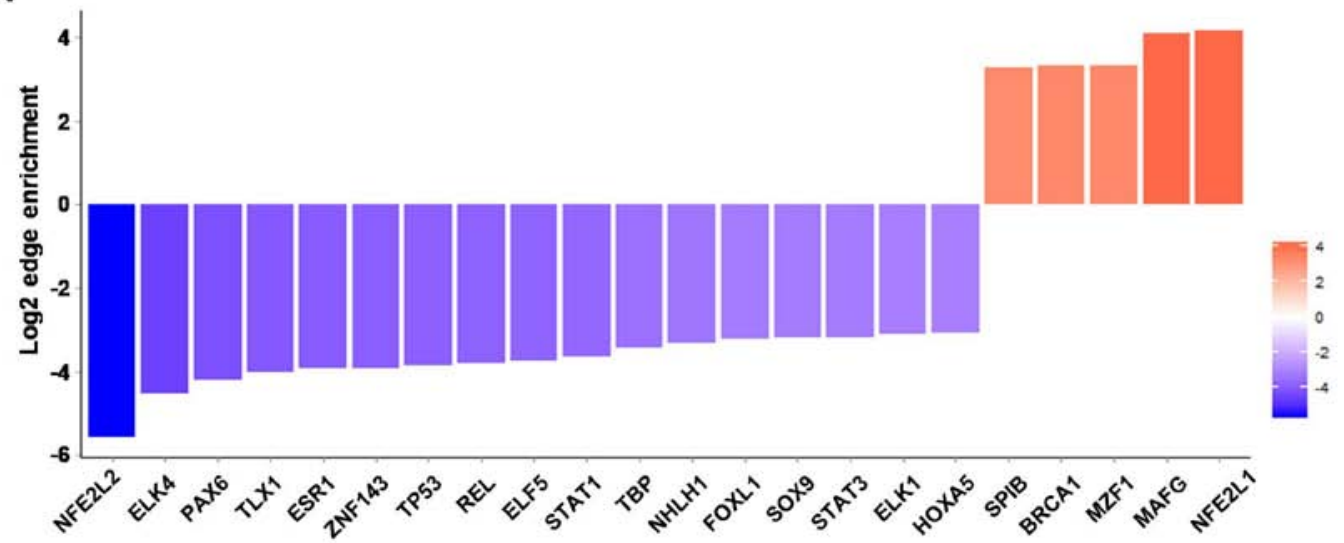

B

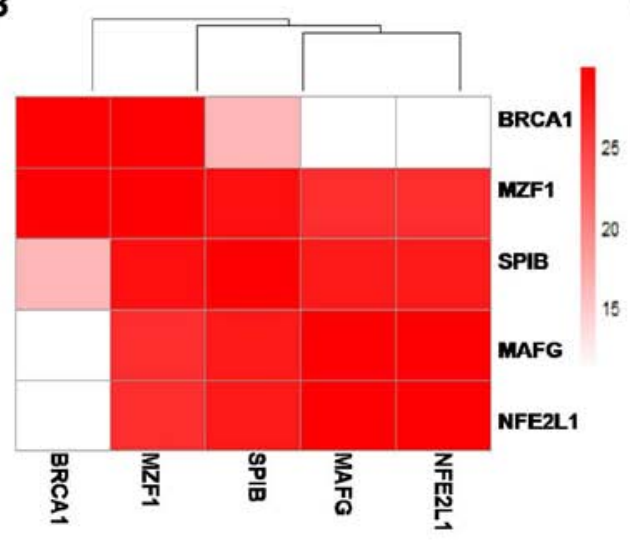

D

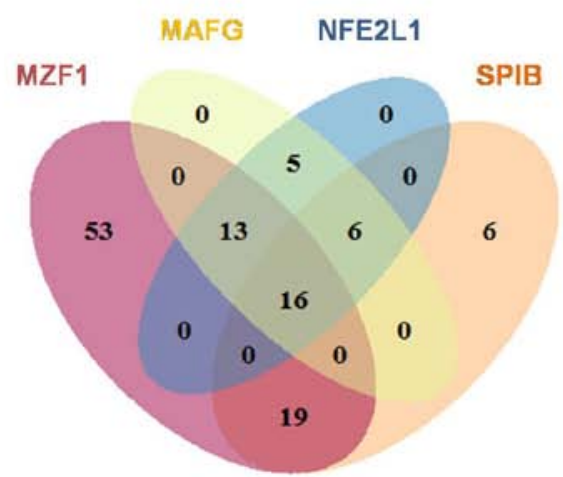

C

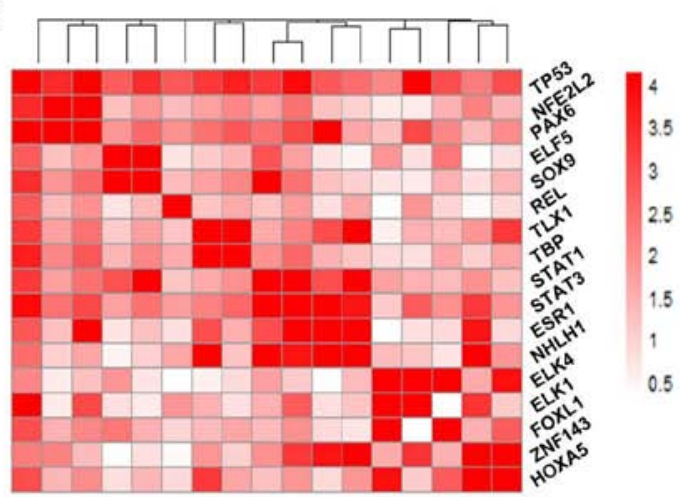

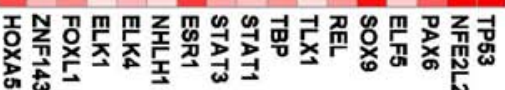

E

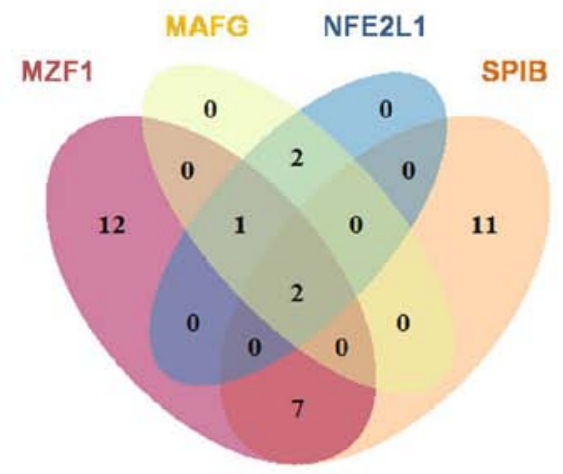

Figure 3. Edge enrichment of transcription factors (TFs) and subsequent co-activation analysis. (A) Edge enrichment of TFs in squamous cell carcinoma (ESCC) by AnaPANDA. (B) Heatmap displayed co-target effects among 5 activated TFs in ESCC. (C) Heatmap displayed co-target effects among 12 activated TFs in non-cancerous (NC) tissues (repressed in ESCC). (D) Venn diagram of target overlapping between SPIB, MZF1, MAFG and NFE2L1 in ESCC-specific network. (E) Venn diagram of target overlapping between SPIB, MZF1, MAFG and NFE2L1 in NC-specific network.

regulatory networks for ESCC and NC were constructed by PANDA integrating gene profiling data with the TF motif data. TF-target edges $(208,887$ and 201,224$)$ were identified in ESCC and NC, respectively, among which 16,970 and 9,307 edges were ESCC- and NC-specific (Fig. 2B-D).

Edge enrichment of TFs in ESCC. In order to find driver TFs which are specifically activated in ESCC, an edge enrichment score and an associated p-value derived from AnaPANDA were generated for each TF, to assess its activity change in ESCC compared to NC. Twenty-two TFs with absolute value of edge enrichment score $>8$ and p-value $<0.05$ were considered activity changed TFs; 17 of them (NFE2L2, ELK4, PAX6, TLX1, ESR1, ZNF143, TP53, REL, ELF5, STAT1, TBP, NHLH1, FOXL1, SOX9, STAT3, ELK1, and HOXA5) were repressed in ESCC and 5 (SPIB, BRCA1, MZF1, MAFG and NFE2L1) were activated in ESCC (Fig. 3A).

Co-activation of 4 TFs were identified in ESCC. To evaluate co-activation and co-repression effect among those TFs, hypergeometric distribution model-based target profile similarity analysis was performed. Only ESCC- and 
A

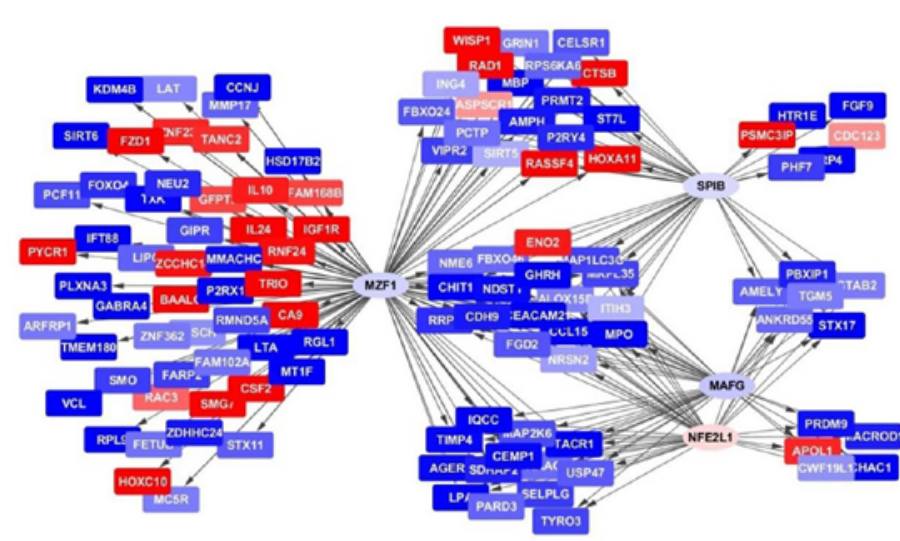

B

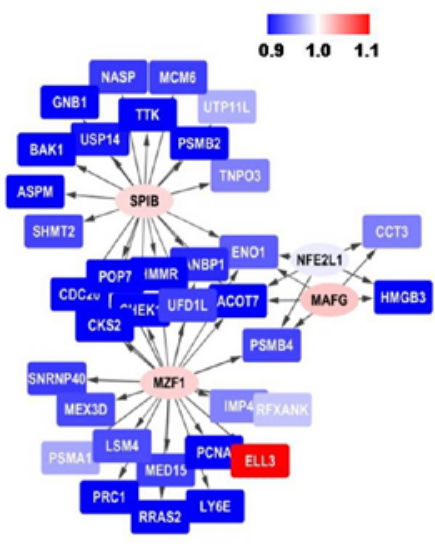

C

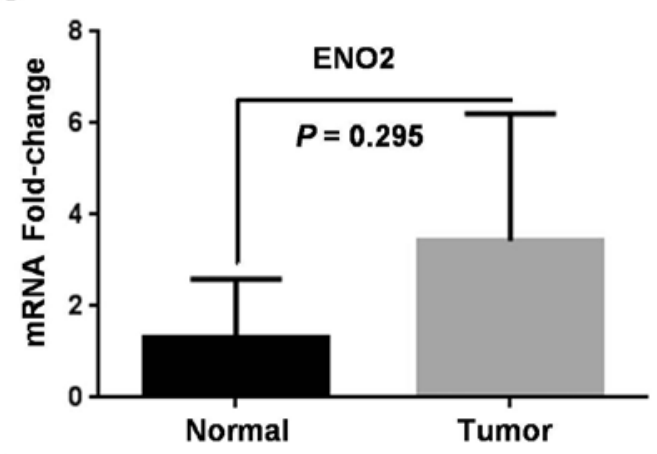

E

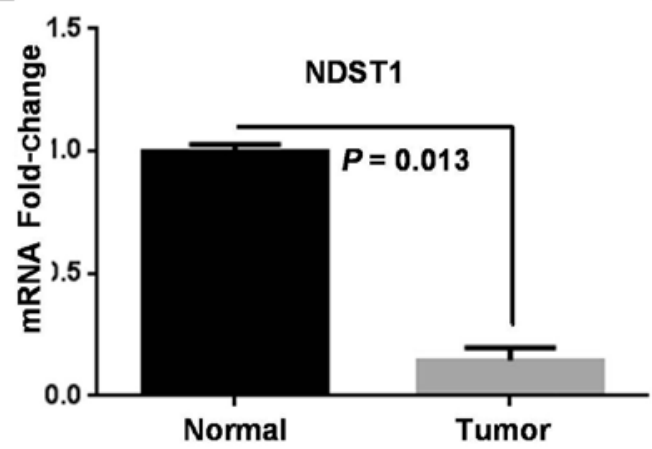

D

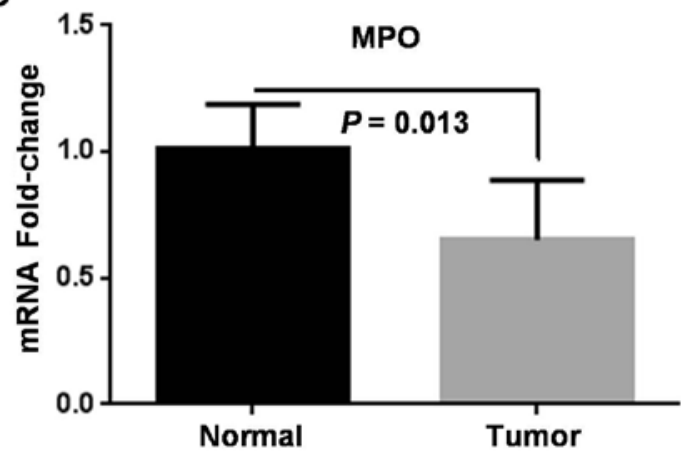

F

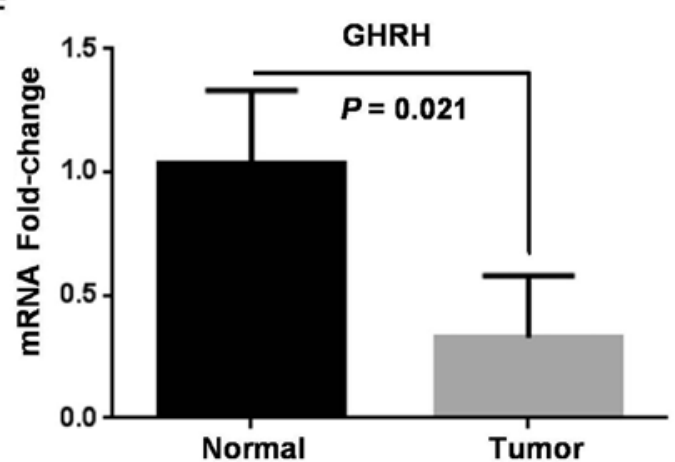

Figure 4. TF-target regulatory networks were different in squamous cell carcinoma (ESCC) and paired normal esophageal tissues for 4 transcription facors (TFs) (SPIB, MZF1, MAFG and NFE2L1). (A) An ESCC-specific TF-target regulatory network derived from 4 TFs (SPIB, MZF1, MAFG and NFE2L1). (B) The parallel NC-specific TF-target regulatory network derived from 4 TFs (SPIB, MZF1, MAFG and NFE2L1). (C) mRNA expression fold-change of ENO2 between ESCC and paired normal esophageal tissue. (D) mRNA expression fold-change of MPO between ESCC and paired normal esophageal tissue. (E) mRNA expression fold-change of NDST1 between ESCC and paired normal esophageal tissue. (F) mRNA expression fold-change of GHRH2 between ESCC and paired normal esophageal tissue.

NC-specific edges and significantly differentially expressed targets were included in this model. SPIB, MZF1, MAFG and NFE2L1 exhibited a strong co-activation effect with each other (Fig. 3B; p-value $<1 \times 10^{-25}$ ). TP53, NFE2L2 and PAXB were found to be moderately co-repressed in ESCC (Fig. 3C; p-value $\left.<1 \times 10^{-4}\right)$. STAT1, STAT3, ESR1 and NHLH1 also were found to be moderately co-repressed in ESCC (Fig. 3C; p-value $\left.<1 \times 10^{-4}\right)$.

Specific TF-target regulatory network was identified in ESCC. To elucidate core ESCC TF-target regulatory network, the overlap of targets among the 4 co-activated TFs in ESCC was further investigated. Among all 118 genes targeted at least by one of SPIB, MZF1, MAFG and NFE2L1, there were
16 genes co-targeted by all the 4 TFs, 19 co-targeted by three, 24 co-targeted by 2 of them in ESCC (Fig. 3D). However, there were only 2 genes co-targeted by all the 4 TFs, 1 co-targeted by three, 9 co-targeted by 2 of them in NC (Fig. 3E). Finally, a network diagram was shown to provide intuitive insight into this ESCC-specific TF-target regulatory network (Fig. 4A and B).

Furthermore, only 2 out of 16 genes co-targeted by the 4 TFs were significantly differentially expressed between stage I/II $(n=5)$ and stage III $(n=3)$ ESCC, and only 1 gene co-targeted by the 4 TFs was significantly differentially expressed between well/moderately $(n=5)$ and poorly $(n=3)$ differentiated ESCC. These results indicated that co-activation of the 4 TFs is not associated with ESCC stage or grade of differentiation. 
To further validate the ESCC-specific network, expression of 4 co-targeted genes of the 4 TFs was quantified by qPCR assays in an independent ESCC cohort. ENO2 was 2 -fold upregulated in ESCC (paired $\mathrm{t}=1.203, \mathrm{p}=0.295$ ), while MPO (paired $\mathrm{t}=8.639, \mathrm{p}=0.013$ ), NDST1 (paired $\mathrm{t}=48.851, \mathrm{p}=0.013$ ), and $\mathrm{CDH} 9$ (paired $\mathrm{t}=4.457, \mathrm{p}=0.021$ ) were significantly downregulated in ESCC (Fig. 4C-F). Expression of all the co-targeted genes was in accordance with their expression in our predicated network.

\section{Discussion}

There have been numerous studies of high-throughput omics analysis in cancer. However, studies focusing on regulatory spectrums are not common. For cancers with a low incidence in developed areas, such as ESCC, transcriptional regulatory network analysis is lacking.

By combining multiple sources of data to model TF-target regulatory networks, integrative analyses showed strong power in the investigation of pathologic mechanisms $(23,24)$. PANDA is a well-established integrative analysis tool which is successfully used to study chronic disease (25) and cancer (20). PANDA predicts TF-gene regulatory relationships based on information from gene expression and TF-sequence-motif data with a message-passing approach. In the present study, we used PANDA and its appended program AnaPANDA to investigate transcriptional regulatory networks in ESCC and NC tissues. We found 1,116 upregulated genes and 1,301 downregulated genes in ESCC and identified 16,970 ESCC-specific TF-target edges and 9,307 NC-specific TF-target edges.

Further edge enrichment analysis found 17 TFs repressed in ESCC and 5 TFs activated in ESCC. Co-activation and co-repression effects were also identified in these TFs, which indicated that the change in transcriptional regulatory networks in ESCC was coupled and not isolated. Four TFs, i.e. SPIB, MZF1, MAFG and NFE2L1, exhibited a strong and significant co-activation effect in ESCC, which was suggested to be an important TF regulatory module in carcinogenesis of ESCC.

MZF-1, a Kruppel family protein, functions as a crucial TF in hematopoietic development (26), which exerts oncogenic or tumor-suppressive functions in various types of cancer. In colorectal cancer (CRC) and gastric cancer (GC), MZF1 was found to increase migration and invasion ability by facilitating transcription of MMP-14 $(27,28)$. However, in cervical cancers, MZF1 repressed expression of MMP2 to exert tumorsuppressive functions (29).

NFE2L1 is a member of the CNC-bZIP family, which has been reported to regulate the mTORC1 signaling pathway and increase cellular proteasome levels. Various studies have shown that NFE2L1 is involved in hypoxia and oxidative stress response of cancer cells $(30,31)$. In ESCC, the role of NFE2L1 is poorly understood, and our results suggest a similar role as that in gastroenterological tumors.

MAFG is a transcription factor of the Maf family proteins (32), which have been reported to regulate bile acid homeostasis (33). MAFG was also found to exert an oncogenic function in hepatocellular carcinoma (HCC) and colorectal cancer, enhance tumor invasiveness by upregulating $\beta$-catenin in $\mathrm{HCC}$ and promote $\mathrm{CpG}$ island methylation in BRAF-mutant colorectal cancer $(34,35)$.
SPIB, known as a member of the Ets family, participates in the differentiation of mature B-cells and plasmacytoid dendritic cells (36-39), which have been mostly reported in hematologic-associated cancers with a role of mediating apoptosis via the PI3K-AKT pathway (40). A gene profiling assay showed that SPIB promotes the progression of gastric cancers (41). Our results also suggested that SPIB is oncogenic in ESCC.

These 4 TFs were found to be oncogenic in tumors of the digestive system, but their co-activation effect was not reported, nor were their effects in ESCC. Our results not only indicate that they have a potential carcinogenic role in ESCC, but also indicate that further investigation may contribute to the further understanding of their co-regulation and co-targeting profile in ESCC.

In conclusion, in the present study, we constructed transcriptional regulatory networks of ESCC and NC tissues. Combining these networks with a gene expressing analysis, we found SPIB, MZF1, MAFG and NFE2L1 co-activated in ESCC, which could be an important co-regulatory mechanism underlying the carcinogenesis of ESCC.

\section{Acknowledgements}

The present study was supported by the National Natural Science Foundation of China (grant nos. 81302160 and 81272447), and the Beijing Natural Science Foundation Program and Scientific Research Key Program of Beijing Municipal Commission of Education (grant no. KZ201410025024).

\section{References}

1. Jemal A, Bray F, Center MM, Ferlay J, Ward E and Forman D: Global cancer statistics. CA Cancer J Clin 61: 69-90, 2011.

2. Zhu HC, Yang X, Xu LP, Zhao LJ, Tao GZ, Zhang C, Qin Q, Cai J, Ma JX, Mao WD, et al: Meat consumption is associated with esophageal cancer risk in a meat- and cancer-histological-type dependent manner. Dig Dis Sci 59: 664-673, 2014.

3. Kamangar F, Dores GM and Anderson WF: Patterns of cancer incidence, mortality, and prevalence across five continents: Defining priorities to reduce cancer disparities in different geographic regions of the world. J Clin Oncol 24: 2137-2150, 2006.

4. Chen M, Huang J, Zhu Z, Zhang J and Li K: Systematic review and meta-analysis of tumor biomarkers in predicting prognosis in esophageal cancer. BMC Cancer 13: 539, 2013.

5. Guo M, Ren J, House MG, Qi Y, Brock MV and Herman JG: Accumulation of promoter methylation suggests epigenetic progression in squamous cell carcinoma of the esophagus. Clin Cancer Res 12: 4515-4522, 2006.

6. Jia Y, Yang Y, Zhan Q, Brock MV, Zheng X, Yu Y, Herman JG and Guo M: Inhibition of SOX17 by microRNA 141 and methylation activates the WNT signaling pathway in esophageal cancer. J Mol Diagn 14: 577-585, 2012.

7. Wu L, Herman JG, Brock MV, Wu K, Mao G, Yan W, Nie Y, Liang $\mathrm{H}$, Zhan $\mathrm{Q}$, Li W, et al: Silencing $D A C H 1$ promotes esophageal cancer growth by inhibiting TGF- $\beta$ signaling. PLoS One 9: e95509, 2014.

8. Guo M, Ren J, Brock MV, Herman JG and Carraway HE: Promoter methylation of HIN-1 in the progression to esophageal squamous cancer. Epigenetics 3: 336-341, 2008.

9. Jia Y, Yang Y, Brock MV, Cao B, Zhan Q, Li Y, Yu Y, Herman JG and Guo M: Methylation of TFPI-2 is an early event of esophageal carcinogenesis. Epigenomics 4: 135-146, 2012.

10. Wang JS, Guo M, Montgomery EA, Thompson RE, Cosby H, Hicks L, Wang S, Herman JG and Canto MI: DNA promoter hypermethylation of $\mathrm{p} 16$ and APC predicts neoplastic progression in Barrett's esophagus. Am J Gastroenterol 104: 2153-2160, 2009. 
11. Yun T, Liu Y, Gao D, Linghu E, Brock MV, Yin D, Zhan Q, Herman JG and Guo M: Methylation of CHFR sensitizes esophageal squamous cell cancer to docetaxel and paclitaxel. genes. Cancer 6: 38-48, 2015.

12. Rong R, Jiang LY, Sheikh MS and Huang Y: Mitotic kinase Aurora-A phosphorylates RASSF1A and modulates RASSF1Amediated microtubule interaction and $\mathrm{M}$-phase cell cycle regulation. Oncogene 26: 7700-7708, 2007.

13. Kuroki T, Trapasso F, Yendamuri S, Matsuyama A, Alder H, Mori M and Croce CM: Promoter hypermethylation of RASSF1A in esophageal squamous cell carcinoma. Clin Cancer Res 9: $1441-1445,2003$

14. Maesawa C, Tamura G, Nishizuka S, Ogasawara S, Ishida K, Terashima M, Sakata K, Sato N, Saito K and Satodate R: Inactivation of the $C D K N 2$ gene by homozygous deletion and de novo methylation is associated with advanced stage esophageal squamous cell carcinoma. Cancer Res 56: 3875-3878, 1996.

15. Lee EJ, Lee BB, Kim JW, Shim YM, Hoseok I, Han J, Cho EY, Park J and Kim DH: Aberrant methylation of Fragile Histidine Triad gene is associated with poor prognosis in early stage esophageal squamous cell carcinoma. Eur J Cancer 42: 972-980, 2006.

16. Barabási AL: Network medicine - from obesity to the 'diseasome'. N Engl J Med 357: 404-407, 2007.

17. Silverman EK and Loscalzo J: Developing new drug treatments in the era of network medicine. Clin Pharmacol Ther 93: 26-28, 2013.

18. Silverman EK and Loscalzo J: Network medicine approaches to the genetics of complex diseases. Discov Med 14: 143-152, 2012

19. Papin JA, Reed JL and Palsson BO: Hierarchical thinking in network biology: The unbiased modularization of biochemical networks. Trends Biochem Sci 29: 641-647, 2004.

20. Glass K, Quackenbush J, Spentzos D, Haibe-Kains B and Yuan GC: A network model for angiogenesis in ovarian cancer. BMC Bioinformatics 16: 115, 2015.

21. Lao T, Glass K, Qiu W, Polverino F, Gupta K, Morrow J, Mancini JD, Vuong L, Perrella MA, Hersh CP, et al: Haploinsufficiency of Hedgehog interacting protein causes increased emphysema induced by cigarette smoke through network rewiring. Genome Med 7: 12, 2015.

22. Xu CQ, Zhu ST, Wang M, Guo SL, Sun XJ, Cheng R, Xing J, Wang WH, Shao LL and Zhang ST: Pathway analysis of differentially expressed genes in human esophageal squamous cell carcinoma. Eur Rev Med Pharmacol Sci 19: 1652-1661, 2015.

23. Banks CA, Lee ZT, Boanca G, Lakshminarasimhan $M$ Groppe BD, Wen Z, Hattem GL, Seidel CW, Florens L and Washburn MP: Controlling for gene expression changes in transcription factor protein networks. Mol Cell Proteomics 13: 1510-1522, 2014

24. Buckingham M and Rigby PW: Gene regulatory networks and transcriptional mechanisms that control myogenesis. Dev Cell 28: 225-238, 2014

25. Glass K, Quackenbush J, Silverman EK, Celli B, Rennard SI, Yuan GC and DeMeo DL: Sexually-dimorphic targeting of functionally-related genes in COPD. BMC Syst Biol 8: 118, 2014
26. Hromas R, Collins SJ, Hickstein D, Raskind W, Deaven LL, O'Hara P, Hagen FS and Kaushansky K: A retinoic acidresponsive human zinc finger gene, $M Z F-1$, preferentially expressed in myeloid cells. J Biol Chem 266: 14183-14187, 1991.

27. Deng Y, Wang J, Wang G, Jin Y, Luo X, Xia X, Gong J and Hu J: p55PIK transcriptionally activated by MZF1 promotes colorectal cancer cell proliferation. Biomed Res Int 2013: 868131, 2013.

28. Zheng L, Jiao W, Mei H, Song H, Li D, Xiang X, Chen Y, Yang F, Li H, Huang K, et al: miRNA-337-3p inhibits gastric cancer progression through repressing myeloid zinc finger 1-facilitated expression of matrix metalloproteinase 14 . Oncotarget 7 : 40314-40328, 2016.

29. Tsai SJ, Hwang JM, Hsieh SC, Ying TH and Hsieh YH: Overexpression of myeloid zinc finger 1 suppresses matrix metalloproteinase-2 expression and reduces invasiveness of $\mathrm{SiHa}$ human cervical cancer cells. Biochem Biophys Res Commun 425: 462-467, 2012.

30. Katsuoka F and Yamamoto M: Small Maf proteins (MafF, MafG, MafK): History, structure and function. Gene 586: 197-205, 2016.

31. Yang YM, Roh YS, Seki E and Maf G: MafG, a novel target of FXR that regulates bile acid homeostasis. Gastroenterology 149: 1981-1983, 2015.

32. Ding X, Yang Y, Han B, Du C, Xu N, Huang H, Cai T, Zhang A, Han ZG, Zhou W, et al: Transcriptomic characterization of hepatocellular carcinoma with CTNNB1 mutation. PLoS One 9: e95307, 2014.

33. No authors listed: MAFG mediates CIMP in $B R A F$-mutant colorectal cancer. Cancer Discov 4: OF11, 2014.

34. Rui L, Schmitz R, Ceribelli M and Staudt LM: Malignant pirates of the immune system. Nat Immunol 12: 933-940, 2011.

35. Lenz G, Wright GW, Emre NC, Kohlhammer H, Dave SS, Davis RE, Carty S, Lam LT, Shaffer AL, Xiao W, et al: Molecular subtypes of diffuse large B-cell lymphoma arise by distinct genetic pathways. Proc Natl Acad Sci USA 105: 13520-13525, 2008.

36. Sasaki I, Hoshino K, Sugiyama T, Yamazaki C, Yano T, Iizuka A, Hemmi H, Tanaka T, Saito M, Sugiyama M, et al: Spi-B is critical for plasmacytoid dendritic cell function and development. Blood 120: 4733-4743, 2012.

37. Schmidlin H, Diehl SA, Nagasawa M, Scheeren FA, Schotte R, Uittenbogaart $\mathrm{CH}$, Spits $\mathrm{H}$ and Blom B: Spi-B inhibits human plasma cell differentiation by repressing BLIMP1 and XBP-1 expression. Blood 112: 1804-1812, 2008.

38. Schotte R, Nagasawa M, Weijer K, Spits H and Blom B: The ETS transcription factor Spi-B is required for human plasmacytoid dendritic cell development. J Exp Med 200: 1503-1509, 2004

39. Takagi Y, Shimada K, Shimada S, Sakamoto A, Naoe T, Nakamura S, Hayakawa F, Tomita A and Kiyoi H: SPIB is a novel prognostic factor in diffuse large B-cell lymphoma that mediates apoptosis via the PI3K-AKT pathway. Cancer Sci 107: $1270-1280,2016$

40. Zhang Y and Manning BD: mTORC1 signaling activates NRF1 to increase cellular proteasome levels. Cell Cycle 14: 2011-2017, 2015.

41. Kim HM, Han JW and Chan JY: Nuclear factor erythroid-2 like 1 (NFE2L1): Structure, function and regulation. Gene 584: $17-25,2016$ 